\title{
The Importance of Superb Microvascular Imaging for the Differentiation of Malignant Breast Lesions from Benign Lesions
}

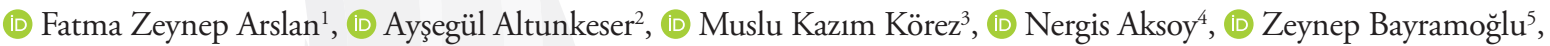
(D) Mehmet Karagülle

${ }^{1}$ Department of Radiology, Basaksehir City Hospital, İstanbul, Turkey

${ }^{2}$ Department of Radiology, University of Health Sciences Turkey, Konya Training and Research Hospital, Konya, Turkey

${ }^{3}$ Department of Biostatistics, Selcuk University Faculty of Medicine, Konya, Turkey

${ }^{4}$ Department of General Surgery, University of Health Sciences Turkey, Konya Training and Research Hospital, Konya, Turkey

${ }^{5}$ Department of Pathology, University of Health Sciences Turkey, Konya Training and Research Hospital, Konya, Turkey

${ }^{6}$ Department of Radiology, University of Health Sciences Turkey, İstanbul Training and Research Hospital, İstanbul, Turkey

\begin{abstract}
Objective: In this prospective study, the diagnostic performance of the new version of superb microvascular imaging (SMI) in differentiating malignant from benign lesions was evaluated.

Material and Methods: Ninety breast lesions were included. During color SMI examination, both free-hand region of interest (ROI) and box ROI were used. Vascular index (VI) values were obtained from the lesion using both types of ROI and from normal breast tissue via box ROI. VI values, monochrome SMI grading and histopathological results were compared. The efficacy of color SMI and monochrome SMI was investigated in differentiating between benign and malignant breast lesions.

Results: The cut-off value, in the differentiation of benign and malignant lesions with color SMI was 0.50 for box ROI, while it was 0.30 for free-hand ROI. The specificity of VI values obtained with box ROI was higher than that of free-hand ROI when differentiating malignant lesions from benign. Comparison of VI values from a lesion and from normal breast tissue showed that VI values in malignant lesions were significantly higher $(\mathrm{p}<0.05)$. The VI values of benign lesions and VI values of normal breast tissue were similar. There was a statistically significant relationship between monochrome SMI grading and the malignancy or benign status of the lesion $(\mathrm{p}<0.001)$.

Conclusion: Drawing the lesion circumference free-hand using a free-shape ROI did not enhance the sensitivity and specificity. Contrary to popular belief, a more easy and practical measurement method may be more suitable for SMI examination. It is hoped that this will be one of the earliest studies to assess the clinical performance of the latest version of SMI.
\end{abstract}

Keywords: Superb microvascular imaging, new version, breast lesions, women's health, ultrasonography

Cite this article as: Arslan FZ, Altunkeser A, Körez MK, Aksoy N, Bayramoğlu Z, Karagülle M. The Importance of Superb Microvascular Imaging for the Differentiation of Malignant Breast Lesions from Benign Lesions. Eur J Breast Health 2022; 18(1): 48-54

\section{Key Points}

- $\quad \mathrm{SMI}$ is a promising development to improve the differentiation of malignant and benign breast lesions because of its superiority in imaging microvascular structures in breast lesions.

- The qualitative and quantitative values obtained from the detailed display of the blood supply in the tumoral tissue can be used as an indirect indicator of abnormal vascularity.

- The vascular index has only been used in a few recent current SMI studies and only a few of these.

- Lesions with a high risk for breast cancer can be easily detected with the contribution of SMI and can also serve as a guide for indeterminant lesions. 


\section{Introduction}

Breast cancer is the most common type of cancer in women and among the most common causes of death $(1,2)$. Advances in tools for early diagnosis contributed significantly to a decrease in the death rate due to breast cancer. It is sometimes challenging to distinguish malignant lesions from benign lesions using radiological imaging methods because of the wide spectrum of imaging and pathological features of breast lesions (3). Malignant breast lesions express high metabolic activity and require newly developed microvascular structures to invade the basement membrane (4). Thus malignant breast lesions exhibit greater vascularity compared to benign lesions and develop irregular vascular structures within the tumor, termed neovascularization (5). To be able to identify this irregular vascularity may increase the diagnostic effectiveness of conventional methods.

Superb microvascular imaging (SMI), an alternative Doppler ultrasonography (US) method developed in recent years using a new adaptive algorithm, separates tissue movements from the slow flow of small vessels and provides novel additional information compared to conventional imaging methods $(3,6)$. In the latest version of SMI, used in the present study, quantitative values for vascularity, known as the vascular index (VI) can be obtained. Thus, it is possible to objectively evaluate the presence of microvascular structures in a selected area of the diagnostic images (7). Given the neovascularity of malignant lesions, we hypothezised that SMI would aid in breast cancer diagnosis.

The aim of this study was to evaluate the vascularity of breast lesions using SMI, and to compare and correlate the findings with histopathological results. In contrast to previous studies, with this newly developed version of SMI, an additional aim was to investigate whether there is an objective quantitative value of VI that can distinguish malignant breast lesions from benign lesions. Thus, it was hoped that this study would be one of the first to provide numerical values obtained with SMI for distinguishing malignant from benign lesions.

\section{Materials and Methods}

Female patients who attended Outpatient Clinic between 01.01.201901.09.2019 and had suspicious breast lesions on US were examined prospectively. Oral and written consent were obtained from all patients who participated in our study. Ethics committee approval was obtained from the Scientific Research Ethics Committee of Selçuk University Faculty of Medicine.

Solid lesions classified as Breast Imaging Reporting and Data Systems (BI-RADS) categories $4 \mathrm{a}, 4 \mathrm{~b}, 4 \mathrm{c}$ and 5 in breast US were eligible for inclusion in the study. Exclusion criteria were: patients with a history of mastectomy; patients with severe organ failure; patients who underwent chemo-radiotherapy; and those without histopathological results reported by our own histopathologists.

US and SMI were performed in the supine position in all patients, with 7-14 MHz high-frequency probes using a US device, the Aplio 300 (Toshiba, Tokyo, Japan). On grayscale US examination, the size of the lesions, contour features, location in relation to the skin, echo pattern and posterior acoustic properties were evaluated. The sonographically detected morphological features of the lesion were categorized according to the BI-RADS categorization, proposed by the American College of Radiology (8).
After sonographic examination, SMI was performed. During the SMI examination, the scale was $1.5-2.5 \mathrm{~cm} / \mathrm{s}$, mechanical index, wall filter, and frame rate were $1.5,1.5,50-100 \mathrm{~Hz}$, and $>50 \mathrm{~Hz}$, respectively. SMI has two different modes, color SMI (cSMI) and monochrome SMI (mSMI). Initial examination was performed with cSMI, during which the "box" region of interest (ROI), a built-in feature of the program, and free-hand ROI, marked by manually drawing around the lesion, were used. VI values were measured using both ROI types. The number of vascular codes was divided by the area of the ROI, and thus VI was calculated automatically by the device. VI values were measured in the range from 0 to 100 . Box ROI and VI values were obtained for normal tissue from the same quadrant of the contralateral breast without lesions. After completing cSMI, mSMI was performed. The skeletal structure of the microvascular vessels was visually evaluated using mSMI. We created a grading system to visually score the vascularity of the lesions for mSMI. Accordingly, Grade 1 was defined as a normal background with punctate blood supply and minimal vascularity. In Grade 2, vascularity was observed in lesions in the absence of anarchic vascular structures and no more than two linear microvascular signals were detected. Lesions with an anarchic blood supply or more than two vascular structures were classified as Grade 3. Observation of distorted, irregularly shaped and curved microvascular structures in the center and periphery of the lesion on mSMI was accepted as an anarchic blood supply. In three cases recommended by the clinician or requested by the patient, and patients with BI-RADS category $4 \mathrm{a}, 4 \mathrm{~b}, 4 \mathrm{c}$ and 5 lesions, tru-cut biopsy was performed after SMI and histopathological results were obtained. In patients whose tru-cut biopsy results were benign, no further surgery was performed and final results were those reported for the tru-cut biopsy. All lesions that were found to be malignant or indeterminate on biopsy were surgically removed (lumpectomy or mastectomy). These lesions were thus definitive surgical results.

\section{Statistical Analysis}

All statistical analysis was performed using $\mathrm{R}$ version 3.6.0 (The $\mathrm{R}$ Foundation for Statistical Computing, Vienna, Austria; https:// www.r-project.org). was used for statistical analysis. Continuous data are presented as mean \pm standard deviation (SD) or median (interquartile range), and categorical data are presented as the number (n) and percentage (\%). The normality of the data was assessed using the Anderson-Darling normality test. Homogeneity of variances was tested using Levene's test. Mann-Whitney U test was used to compare the VI values obtained with cSMI in malignant and benign lesions. A $p<0.05$ was assumed to indicate significance. The diagnostic performance of the VI values obtained by box and free-hand ROIs in distinguishing malignant and benign lesions was calculated. The cut-off values for distinguishing malignant and benign lesions were determined using receiver operating characteristic (ROC) curves, and performance was evaluated. For the cut-off value, sensitivity, specificity, positive and negative predictive values, and accuracy were calculated at a $95 \%$ confidence level. Interactive point charts were created to determine the threshold values. The relationship between VI values of benign or malignant lesions and VI values of normal tissue was tested using Spearman's rho correlation coefficient. The relationship between mSMI grading and pathological findings was evaluated using the chisquare test.

\section{Results}

Eighty-six women with lesions assessed bu US imaging to be at risk of malignancy were included in the study. Lesions in four patients were 
bilateral. The mean \pm SD diameter of 90 lesions detected on US was $21.89 \pm 17.12 \mathrm{~mm}$, and 49 lesions were located in the right breast. The majority of lesions were BI-RADS category $4 \mathrm{~b}$ and numbered 32 (35.6\%). There were a further $19(21.1 \%)$ of lesions classified as BIRADS category 5 (Table 1 ).

Thirty breast lesions were malignant, and 60 were benign on histopathological examination. The majority of malignant lesions were invasive ductal carcinomas $(\mathrm{n}=28,93.3 \%)$. One patient had invasive lobular carcinoma, and one patient had ductal carcinoma in situ. Fibroadenomas constituted the majority of benign lesions.

For benign lesions, the mean VI value was 1.68 for the box ROI and 0.81 for free-hand ROI (Table 2). While the mean VI value measured using box ROI in malignant lesions was 4.30, the mean free-shaped ROI was found to be 3.23 (Table 2). The VI values of benign and malignant lesions measured by SMI were statistically significant for the both ROI type $(\mathrm{p}<0.001)$.

The cut-off VI value for cSMI, was 0.50 and above for box ROI, while it was 0.30 and above for free-hand ROI. While the sensitivity of VI value measured by box ROI to differentiate benign lesions from malignant lesions was $89 \%$ and the specificity was $56 \%$, these values for free-hand ROI were $89 \%$ and $49 \%$, respectively. The NPV was $92 \%$ for the box ROI and 91\% for free-hand ROI while the PPV for box ROI was $46 \%$ and $43 \%$ for free-hand ROI (Table 3; Figures 1 and 2).

The area under the ROC curve showed that SMI gave significant results in distinguishing between malignant and benign lesions (Graph 1). Differential and interactive point charts were drawn for box and free-hand cSMI VI values, which were used to distinguish between benign and malignant lesions (Graph 2, Table 4).

In the comparison of VI values of the lesion and normal breast tissue, box and free ROI VI values detected in malignant lesions were significantly higher than those in normal breast tissue $(\mathrm{p}<0.05)$. There was no statistically significant difference between the VI values found in benign lesions and VI values obtained from normal breast tissue (p>0.05) (Table 4).

Table 1. Descriptive Features

\section{Parameters}

\section{Age (year), mean, (min-max) \\ US diameter (mm), mean, (min-max)}

Side

Left breast, $\mathrm{n}$

Right breast, $n$

\section{US BIRADS}

BI-RADS 3, n

BI-RADS 4a, $n$

BI-RADS $4 b, n$

BI-RADS 4c, n

BI-RADS 5, n

US: Ultrasonography, BI-RADS: Breast Imaging Reporting and Data Systems, min: Minimum, max: Maximum, n: number
There was a statistically significant correlation between the mSMI grading and whether the lesion was malignant or benign (pathological finding) $(\mathrm{p}<0.001)$. While $94.4 \%$ of the lesions with Grade 1 vascularity were benign, $76.0 \%$ of the Grade 3 lesions were malignant. As the severity (grade) of vascularity detected with mSMI increased, the rate of malignancy increased. However, $5.6 \%$ of the lesions with Grade 1 vascularity were malignant, and $24.0 \%$ of the Grade 3 lesions were benign (Table 5).

\section{Discussion and Conclusion}

US is the basic imaging modality used for examination of dense breasts with a high degree of fibroglandular tissue components (9). However, descriptive morphological features, such as margin, shape or echo pattern of the lesion do not always provide clear

\section{Table 2. Comparison of vascular index (VI) values of benign} and malignant lesions measured by SMI using two modes for defining regions of interest (ROI), box ROI and free-hand ROI

\begin{tabular}{lcc}
\hline \multicolumn{2}{c}{ VI value } \\
& cSMI box & cSMI free \\
\hline $\begin{array}{l}\text { Benign ( } n=63) \\
\text { mean } \pm \text { SD }\end{array}$ & $1.68 \pm 3.21$ & $0.81 \pm 1.44$ \\
Malign ( $n=27)$ & & \\
mean \pm SD & $4.30 \pm 5.51$ & $3.23 \pm 4.41$ \\
* $p$-value & $<0.001$ & $<0.001$ \\
\hline
\end{tabular}

ROI: region of interest, n: Number of lesions, CSMI box: Box shape ROI. CSMG free: free-hand ROI. SD: Standard deviation

${ }^{*} p<0.05$ was considered as statistically significant. Mann Whitney-U was used.

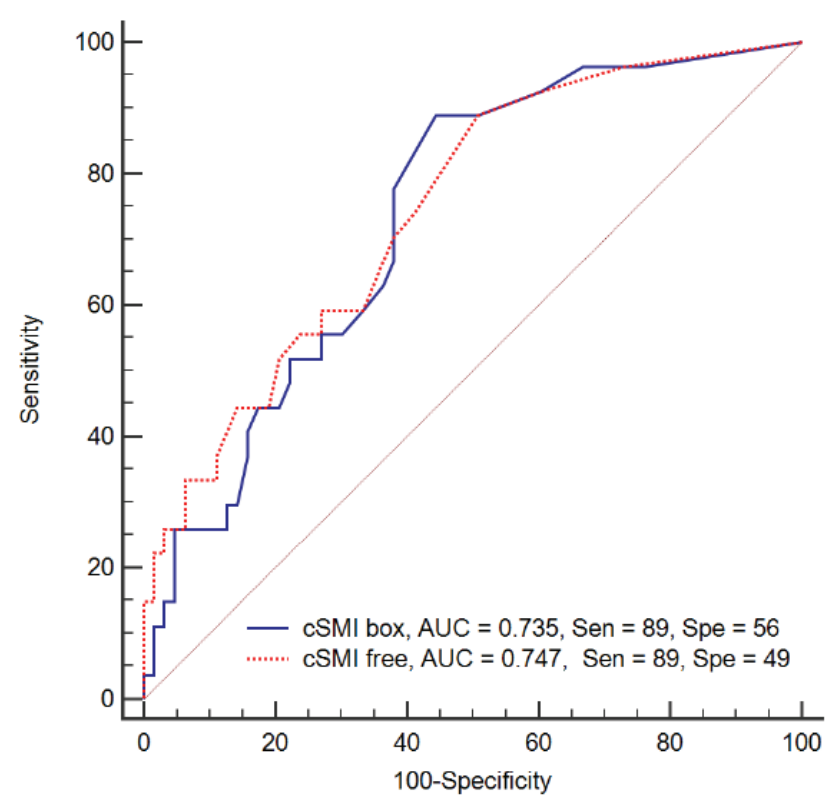

Graph 1. ROC curve for box and free cSMI VI value used in differentiation of benign and malignant lesions

CSMI: Color superb microvascular imaging, CSMI box: Box shaped ROI, CSMI free: Free shaped ROI, ROC: Receiver operating characteristics, Sen: Sensitivity. Spe: Specificity 
Table 3. Diagnostic effectiveness of box and free cSMI in differentiating benign and malignant lesions

\section{cSMI VI value}

Box

Free-hand

\section{Diagnostic measurements (\%)}

Cut-off value

$\geq 0.50$

$\geq 0.30$

TP-FP-FN-TN

24-28-3-35

24-32-3-31

(subsquently)

Sensitivity

89

89

Specificity

56

49

NPV

92

91

PPV

46

43

\section{ROC statistics}

AUC

0.735

0.747

${ }^{*} \mathrm{p}$-value

$<0.001$

$<0.001$

CSMI: Color superb microvascular imaging, VI: Vascularity index, ROI: region of interest, Box: Box shaped ROI, Free-hand: Free-hand drawn ROI, TP: True positive value, FP: False positive value, FN: False negative, TN: True negative, NPV: Negative predictive value; PPV: Positive predictive value, ROC: Receiver operating characteristics curve, AUC: Area under the ROC curve

${ }^{*} \mathrm{p}<0.05$ was considered statistically significant. Mann-Whitney $U$ test was used.
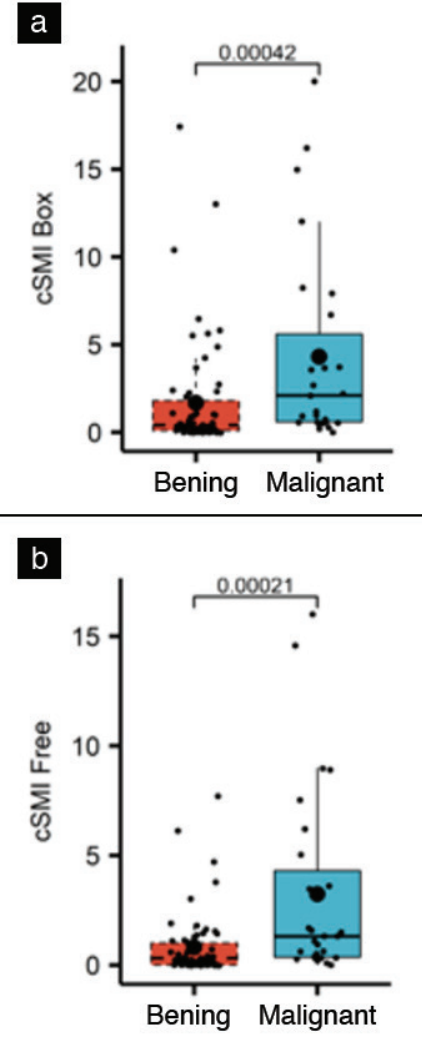

Graph 2. Difference and interactive point graphic for VI value detected by CSMI in benign and malignant lesions

CSMI: Color superb microvascular imaging, VI: Vascular index, CSMI box: box shaped ROI, cSMIfree: Free-hand drawn ROI information about whether the lesion is malignant or benign. In particular, granulomatous mastitis, atypical hamartoma and some fibroadenomas do not exhibit the characteristics of typical BI-RADS category 3 (10). DM is the most important radiological screening and diagnostic tool that has been proven to increase survival in breast cancer (11). While DM has great sensitivity in fatty breasts, it decreases to $30 \%$ in dense breasts. Especially in young patients, DM cannot provide very detailed information and when these patient groups are considered, alternative methods are needed to increase the effectiveness of US (12). Breast MRI has the highest sensitivity for distinguishing breast cancer among the available modern imaging modalities (13). However, performing MRI on every patient is timeconsuming and not cost-effective.

Conventional sonographic methods, such as color and power Doppler do not provide the necessary additional information in such breast lesions. Thus, unnecessary biopsies and surgical procedures may be performed. In breast cancer, angiogenic factors and abnormal neovascular vessels develop within the tumoral tissue. For this reason, distorted, folded and deeply penetrating vascular structures are observed around tumoral lesions. Regularly shaped microvessels are observed in benign lesions (14). Thus, attempts have been made to reveal irregular neovascular vessels using complementary methods in addition to US. Conventionally, color Doppler imaging and power Doppler imaging are used to show tissue vascularity. Unfortunately, classical Doppler methods identify the slow flow of microvascular structures as artifacts and therefore erase them $(14,15)$.

Table 4. Relationship between VI values of benign and malignant lesions and VI values of normal tissue

\begin{tabular}{lcccc} 
& \multicolumn{2}{c}{$\begin{array}{c}\text { Comparison of } \\
\text { benign lesion and } \\
\text { normal breast } \\
\text { tissue }\end{array}$} & $\begin{array}{c}\text { Comparison of } \\
\text { malignant lesion } \\
\text { and normal breast } \\
\text { tissue }\end{array}$ \\
cSMI box & p & P & P & P \\
cSMI free & 0.095 & 0.458 & $0.478^{*}$ & 0.012 \\
& 0.210 & 0.098 & $0.468^{*}$ & 0.014
\end{tabular}

cSMI: Color superb microvascular imaging, VI: Vascularity index, $\mathrm{p}$ : Spearman's rho correlation coefficient

${ }^{*} p<0.05$ was considered as statistically significant

Table 5. The relationship between monochrome SMI grading and pathological finding

$\begin{array}{lcccc}\text { mSMI Grade } & \text { Pathological finding } & \begin{array}{c}\text { Total } \\ \text { lesion (n) }\end{array} & \text { p-value } \\ & \text { Benign } & \text { Malign } & & \\ \text { Grade } 1, n & 51 & 3 & 54 & \\ \text { Grade 2, n } & 6 & 5 & 11 & \\ \text { Grade 3, n } & 6 & 19 & 25 & <0.001 \text { * } \\ \text { Total lesion, n } & 63 & 27 & 90 & \end{array}$

SMI: Superb microvascular imaging, n: Number of lesions ${ }^{*} p<0.05$ was considered statistically significant. Chi-square test was used 

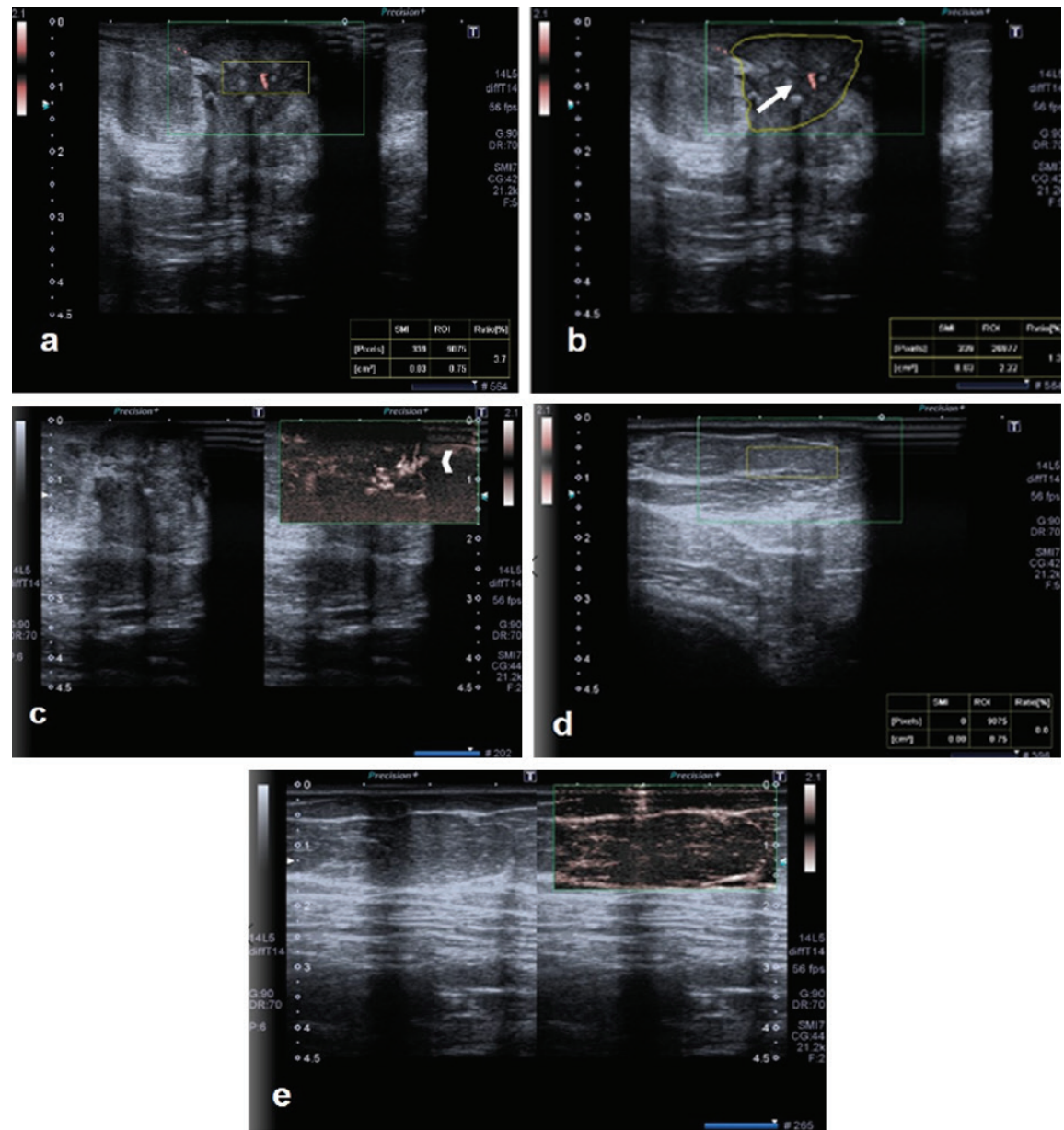

Figure 1. A lesion on the left breast at the 3 o'clock position, vertically located in relation to the skin and with irregular contours, and subsequently, histopathologically proven as invasive ductal carcinoma, is shown. On cSMG examination: a) the VI value obtained from inside the lesion using box ROI was 3.7; b) the value obtained by using free ROI was 1.3. (arrow); c) on mSMG examination, both peripherally and centrally located, irregularly-shaped, microvascular structures are observed (arrowhead). d) For normal breast tissue; e) the VI values taken with the box ROI are zero

SMG: scintimammography, VI: Vascular index, SMI: Superb microvascular imaging, ROI: region of interest
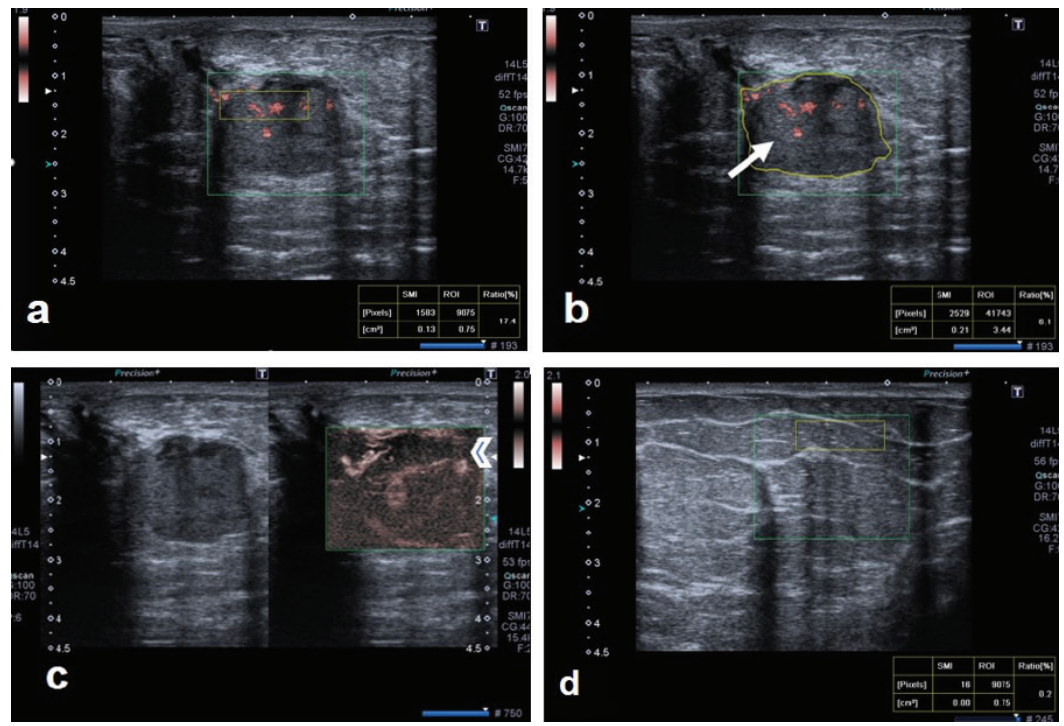

Figure 2. A round-shaped breast lesion, diagnosed as papillary neoplasia after biopsy, at the 9 o'clock position in the left breast. On cSMG examination: a) the VI value obtained from inside the lesion using box ROI was 17.4 ; b) the value obtained by using free ROI was 6.1 (arrow). c) On mSMI examination, both peripheral and centrally located, irregularly-shaped, microvascular structures were observed (arrowhead). d) Box ROI VI value for normal tissue from the same quadrant of the contralateral breast was 0.2 . 
While traditional Doppler methods are successful in showing strong flows with high velocity, they are insufficient for showing the slow flow of small vessels (16). The macrovascular structure of the lesion is evident with color Doppler US, while the microvascular structures, found histopathologically, are not visible. Many recent studies have shown that blood supply within the lesion, detected by color and power Doppler US, cannot distinguish benign from malignant lesions (17). It has been shown that the highest diagnostic accuracy is obtained by combining SMI examination with gray-scale US to identify tumor microvasculature (18). Unlike other conventional Doppler methods, the addition of adaptive software in SMI distinguishes the slow flow of microvascular structures from artifact created by tissue movement. However, VI has only been used in a small number of recent SMI studies and in only a few of these the new version of VI, which gives a quantitative value, was used (19-21). In the present study, VI per unit area was measured using SMI, which is superior to conventional Doppler in showing the slow blood flow of microvessels and demonstrated vascularization in tumoral tissue. In a recent study, cSMI values in malignant lesions were reported to be two-fold higher than those in benign lesions (22). In our study, cSMI values were more than two-and-a-half times greater in malignant compared to benign lesions $(\mathrm{p}<0.001)$. In the present study, specificity for identification of malignant lesions through evidence of irregular vascularity using box ROI was greater than for free-hand ROI and that drawing the lesion circumference by hand using a free-shape ROI did not contribute to determining the vascularity of the lesion. A recent study used a free-hand ROI and the latest version of VI (23). However, in light of our results, drawing lesion contours manually does not appear to contribute any additional data about the nature of the lesion and is more time-consuming. Thus, simply adopting the built-in box ROI feature of the SMI software may be easier and more practical for SMI examination.

A recent study reported that SMI distinguishes benign breast lesions from malignant lesions with $78 \%$ sensitivity and $75 \%$ specificity (17). Bakdik et al. (5) determined the sensitivity and specificity of SMI to be $66.6 \%$ and $80.7 \%$, in a prospective study respectively, in distinguishing malignant intraductal breast lesions. They evaluated a total of 54 intraductal breast lesions and vascularity grading, distribution of microvessels, and penetrating vessels were investigated. Du et al. (18) found that the sensitivity, specificity, and true positivity rates were $93.8 \%, 86.2 \%$, and $90.2 \%$, respectively. In our study, while the sensitivity was $89 \%$ for both ROI types, the specificity was only $56 \%$ for box ROI and $49 \%$ for free-hand ROI, indicating that SMI has a low ability to discriminate benign lesions in our cohort. In contrast to the limited number of published studies, the specificity of SMI was low in our study, and larger-scale prospective studies are needed to elucidate this issue. Based on SMI findings, Bakdik et al. (5) classified the vascularity of the lesions as low, medium and high. They argued that when distinguishing benign and malignant breast lesions with SMI, the highest success is achieved when vascularity is classified as low or high. Bakdık et al. (5) determined the VI cut-off value to be 0.80 to categorize a breast lesion as hypervascular. In our study with a higher number of patients, the VI cut-off value was 0.73 for box ROI and 0.74 for free-hand ROI. Zhan et al. (24) reported that a VI cut-off value of 0.91 was reliable in distinguishing malignant breast lesions. In our study, the VI value of malignant lesions was higher than that of both benign lesions and normal breast tissue, while, interestingly, the VI values of benign lesions and the VI values of normal breast tissue were similar. Our results show that the cut- off value, which demonstrates the diagnostic efficiency of cSMI in the differentiation of benign and malignant lesions was $\geq 0.50$ for box ROI, while it was $\geq 0.30$ and for free-hand ROI. We believe that these differences suggest that the cutoff values should be calculated for each ROI type.

SMI has two different modes, cSMI and mSMI. Similar to existing studies (16), in our study, while quantitative values of intralesional vascularity were obtained with cSMI, more detailed information was obtained about the morphology of microvascular structures with mSMI. Park et al. (25) reported that detailed examination of microvascular structures with mSMI without contrast agent injection increased the diagnostic performance of US. Another study emphasized that most published studies were based on quantitative measurements using cSMI, and few studies have examined microvascular structures with mSMI (18). In our study, including both qualitative and quantitative evaluations, a relationship was found between the irregular vascularity detected on mSMI and the malignancy of the lesion. We found that as the grade of vascularity detected with $\mathrm{mSMI}$ increased, the rate of malignancy increased. However, there was still a degree of false negativity with $5 \%$ of the lesions with Grade 1 vascularity being malignant, and $24 \%$ of Grade 3 lesions being benign. Studies have shown that microvessels in malignant lesions are tortuous and show irregular and chaotic vascularity $(18,26)$. Raza et al. (26) reported that small vascular structures in malignant lesions mostly progressed to penetrate deep into the lesion. Moreover, they emphasized the importance of penetrating small vascular structures by stating that they may be the most important clue for malignancy (26). In a recent study, showing penetration of small vessels were more accurate on SMI compared toclassical Doppler methods (24). In addition, it has been reported that there is a decrease in inter-observer variability compared to classical Doppler methods in the detection of penetrating distorted small vessel structures and vascularity assessment. Park et al. $(22,25)$ reported that if SMI is integrated into the US, SMI decreases the risk level of BI-RADS categories in a significant number of patients and protects patients from unnecessary invasive procedures. As malignant lesions are growing rapidly, necrotic areas may occur within the lesion if the tumoral microvasculature cannot develop sufficiently to feed it. Recent studies have reported that false-negative results can also be obtained in malignant lesions because signals cannot be received with Doppler and SMI from necrotic areas (18). In our study, the false negativity rate of SMI in detecting malignant lesions was found to be $3 \%$.

Our study has some limitations. First, the number of patients included in the study was relatively small. Another limitation was that all patients were evaluated with SMI by a single radiologist and interobserver variability could not be evaluated. In addition, a further significant limitation was the lack of inclusion of MG data and different imaging modalities. Future prospective studies should seek to negate these limitations in their design.

In conclusion, SMI is a promising development to improve the differentiation of malignant and benign breast lesions because of its superiority in imaging microvascular structures in breast lesions. The qualitative and quantitative values obtained from the detailed display of the blood supply in the tumoral tissue can be used as an indirect indicator of abnormal vascularity. Thus, lesions with a high risk for breast cancer can be easily detected with the contribution of SMI and can also serve as a guide for indeterminant lesions. However, prospective studies with larger sample sizes and including comparison 
with different modalities are needed to evaluate the effectiveness of SMI in diagnosis of breast lesions.

Ethics Committee Approval: Ethics committee approval was obtained from the Scientific Research Ethics Committee of Selçuk University Faculty of Medicine (decision number: 2018/400, date: 21.11.2018, number of meetings: 2018/22).

Informed Consent: Informed, written consent was obtained from each patient.

Peer-review: Externally peer-reviewed.

\section{Authorship Contributions}

Surgical and Medical Practices: F.Z.A., A.A.; Concept: F.Z.A., A.A.; Design: F.Z.A., N.A.; Data Collection and/or Processing: F.Z.A., N.A., M.K., M.K.K., Z.B.; Analysis and/or Interpretation: F.Z.A.; Literature Search: F.Z.A.; Writing: F.Z.A.

Conflict of Interest: No conflict of interest declared by the authors.

Financial Disclosure: The authors declare that this study received no financial disclosure.

\section{References}

1. Hendrick RE, Smith RA, Rutledge JH, Smart CR. Benefit of screening mammography in women aged 40-49: a new meta-analysis of randomized controlled trials. J Natl Cancer Inst Monogr 1997; 22: 87. [Crossref]

2. Martin FT, O'Fearraigh C, Hanley C, Curran C, Sweeney KJ, Kerin MJ. The prognostic significance of nodal ratio on breast cancer recurrence and its potential for incorporation in a new prognostic index. Breast J 2013; 19: 388-393. (PMID: 23721403) [Crossref]

3. Fox SB, Generali DG, Harris AL. Breast tumour angiogenesis. Breast Cancer Res 2007; 9: 216. (PMID: 18190723) [Crossref]

4. Stanzani D, Chala LF, Barros Nd, Cerri GG, Chammas MC. Can Doppler or contrast-enhanced ultrasound analysis add diagnostically important information about the nature of breast lesions? Clinics 2014; 69: 87-92. (PMID: 24519198) [Crossref]

5. Bakdik S, Arslan S, Oncu F, Durmaz MS, Altunkeser A, Eryilmaz MA. Effectiveness of Superb Microvascular Imaging for the differentiation of intraductal breast lesions. Med Ultrason 2018; 20: 306-312. (PMID: 30167583) [Crossref]

6. Machado P, Segal S, Lyshchik A, Forsberg F. A Novel Microvascular Flow Technique: Initial Results in Thyroids. Ultrasound Q 2016; 32: 67-74. (PMID: 25900162) [Crossref]

7. Kono T, Kazutoshi F, Gen N. Superb Micro-Vascular Imaging (SMI): clinical advantages of a novel us flow technique in pe- diatric diagnostic Imaging. AOSPR 2017; 4: 18-23. [Crossref]

8. D’Orsi CJ, Sickles EA, Mendelson EB, Morris EA. ACR BI-RADS Atlas, Breast Imaging Reporting and Data System. Reston, VA, American College of Radiology; 2013[Crossref]

9. Shah G, Jankharia B. Pictorial essay: Breast US. Indian J Radiol Imaging 2010; 20: 98-104. (PMID: 20607018) [Crossref]

10. Altunkeser A, Arslan FZ, Eryllmaz MA. Magnetic resonance imaging findings of idiopathic granulomatous mastitis: can it be an indirect sign of treatment success or fail? BMC Med Imaging 2019; 19: 94. (PMID: 31842782) [Crossref]

11. Garcia M, Jemal AW, Ward EM, Center MM, Hao Y, Siegel RL et al; American Cancer Society. Breast Cancer Facts \& Figures 2007-2008. 2007 [Crossref]

12. Mohindra N, Neyaz Z, Agrawal V, Agarwal G, Mishra P. Impact of addition of digital breast tomosynthesis to digital mammography in lesion characterization in breast cancer patients. Int J App Basic Med Res 2018; 8: 33-37. (PMID: 29552533) [Crossref]

13. Mann RM, Cho N, Moy L. Breast MRI: state of the art. Radiology 2019; 292: 520-536. (PMID: 31361209) [Crossref]

14. Xiao XY, Chen X, Guan XF, Wu H, Qin W, Luo BM. Superb microvascular imaging in diagnosis of breast lesions: a comparative study with contrastenhanced ultrasonographic microvascular imaging. Br J Radiol 2016; 89: 20160546. doi: 10.1259/bjr.20160546. (PMID: 27529640) [Crossref]

15. McNicholas MM, Mercer PM, Miller JC, McDermott EW, O'Higgins NJ, MacErlean DP. Color Doppler sonography in the evaluation of palpable breast masses. AJR Am J Roentgenol 1993; 161: 765-771. [Crossref]

16. Machado P, Segal S, Lyshchik A, Forsberg F. A novel microvascular flow technique: initial results in thyroids. Ultrasound Q 2016; 32: 67-74. (PMID: 25900162) [Crossref]

17. Ma Y, Li G, Li J, Ren WD. The diagnostic value of superb microvascular imaging (SMI) in detecting blood flow signals of breast lesions: a preliminary study comparing SMI to color doppler flow imaging. Medicine (Baltimore) 2015; 94: e1502. doi: 10.1097/MD.0000000000001502. (PMID: 26356718) [Crossref]

18. Du J, Li FH, Fang H, Xia JG, Zhu CX. Microvascular architecture of breast lesions: evaluation with contrast-enhanced ultrasonographic micro flow imaging. J Ultrasound Med 2008; 27: 833-42; quiz 844. (PMID: 18499843) [Crossref]

19. Keçeli M, Keskin Z, Keskin S. Comparison of superb microvascular Imaging with other doppler methods in assessment of testicular vascularity in cryptorchidism. Ultrasound Q 2020; 36: 363-370. (PMID: 32956243) [Crossref]

20. Kılınçer A, Durmaz MS, Kıraç CO, Baldane S, Ateş F, Batur A. Evaluation of parenchymal vascularity of the thyroid gland with vascularization index by color superb microvascular imaging in patients with Graves' disease. J Ultrason 2021; 21: 41-47. (PMID: 33796339) [Crossref]

21. Chae EY, Yoon GY, Cha JH, Shin HJ, Choi WJ, Kim HH. Added value of the vascular index on superb microvascular imaging for the evaluation of breast masses: comparison with grayscale ultrasound. J Ultrasound Med 2021; 40: 715-723. (PMID: 32815564) [Crossref]

22. Park AY, Kwon M, Woo OH, Cho KR, Park EK, Cha SH, et al. A Prospective study on the value of ultrasound microflow assessment to distinguish malignant from benign solid breast masses: association between ultrasound parameters and histologic microvessel densities. Korean J Radiol 2019; 20: 759-772. (PMID: 30993927) [Crossref]

23. Durmaz MS, Kara Gedik G, Batur A, Yılmaz F. Using 2-dimensional color superb microvascular imaging vascularization index technique in the assessment of thyroid surgical bed. Med Ultrason 2021; 23: 289-296. (PMID: 33793695) [Crossref]

24. Zhan J, Diao XH, Jin JM, Chen L, Chen Y. Superb microvascular Imaging - a new vascular detecting ultrasonographic technique for avascular breast masses: a preliminary study. Eur J Radiol 2016; 85: 915921.(PMID: 27130051) [Crossref]

25. Park AY, Seo BK. Up-to-date Doppler techniques for breast tumor vascularity: superb microvascular imaging and contrast-enhanced ultrasound. Ultrasonography 2018; 37: 98-106. (PMID: 29025210) [Crossref]

26. Raza S, Baum JK. Solid breast lesions: evaluation with power Doppler US. Radiology 1997; 203: 164-168. (PMID: 9122386) [Crossref] 